\title{
10
}

\section{Not-for-Profit Accountability: Addressing potential barriers}

\author{
Dale Tweedie
}

\section{Introduction}

This chapter analyses barriers that not-for-profit (NFP) organisations face in meeting public demands to be accountable and considers possible responses, based on a study of NFP staff, directors and regulators in Australia. While 'accountability' has many possible meanings, the chapter focuses on barriers to NFPs being accountable for the quality or impact of their services even when they are motivated to do so. Of course, there are many other ways that accountability can break down, including through conflicts of interest and outright fraud. However, given the complexity of accountability demands, there are numerous obstacles to being accountable even with the best intentions. A key finding of this study is that many NFP staff and directors want to be accountable, but encounter various barriers to doing so. The chapter explores three such barriers: 1) reporting

1 Thank you to all participants in this study for their time, trust and insights. Thank you also to Dr Karina Luzia for her collaborative work in conducting the research on which this chapter draws, and to Professor Nonna Martinov-Bennie for her support for, and contributions to, this research agenda. 
that does not improve service quality; 2) NFP boards that lack active members; and 3) red tape reduction that might threaten minimum service standards.

The chapter has three main sections. Section one briefly outlines the research approach and method, which include in-depth interviews with 23 staff, directors and regulators in the Australian NFP sector. Section two summarises the barriers to accountability that emerged from these interviews and analyses how each barrier can impede even well-motivated NFPs' capacity to be accountable. Section three explores potential responses to each barrier, drawing on both NFPs' experiences and relevant research. The chapter particularly emphasises how accountability entails not only formal reporting and policy, but also effective communication. Consequently, improving accountability may require changes to reporting and governance processes and the relationships in which these processes are set.

\section{Research approach and method}

NFPs face growing public demands to be accountable (Productivity Commission 2010: xxx; Treasury 2011), but what accountability means is increasingly complex and unclear. Accountability was once almost synonymous with financial reporting (Gray 1983; Carnegie and Wolnizer 1996); however, its meaning has expanded to encompass a much wider set of expectations about how organisations plan, assess and justify their activities. Academic research typically defines accountability very broadly as the 'giving and demanding for reasons for conduct' (Roberts and Scapens 1985; Sinclair 1995; Unerman and O'Dwyer 2006) or, more strongly, as 'the process of holding actors responsible for actions' (Ebrahim 2003: 814; Fox and Brown 1998: 12; see also Stewart 1984). So defined, accountability extends from traditional governance concerns about board structure and financial reporting and controls to demonstrating effective use of public funds. While there is widespread consensus that NFPs need to be accountable, there is little consensus on which accountability practices are most important and, therefore, what accountable NFP organisations should look like in practice. 
This chapter analyses one aspect of accountability - accountability for service quality - based on a 2014 study of the demands NFPs face to be accountable and how NFP staff and stakeholders are managing these demands. The study conducted in-depth interviews with 23 NFP staff, directors and regulators, who occupied roles at 30 different NFPs at the time of the study. Participants were drawn from all organisational levels, including: four directors in their primary NFP role (nine directors of any NFP); five chief executive officers (CEOs) (or equivalent); ${ }^{2}$ four managers; four frontline service workers; four regulators; and two participants who preferred their primary roles to remain anonymous. A cross-section of participants was selected on the basis that accountability demands are not limited to CEOs and boards, but extend across organisational hierarchies - for example, 'downward accountability' (Edwards and Hulme 1996: 967) of service staff to clients. Since no funders were interviewed, the analysis of the NFP-funder relationship is necessarily one-sided. Nonetheless, understanding NFPs' perspectives on this relationship provides an important, albeit partial, perspective. The majority of participants (14, or 61 per cent) worked at large NFPs (more than \$1 million in revenue); three participants (13 per cent) worked at medium-sized NFPs (\$250,000 - \$1 million in revenue); three (13 per cent) were regulators; and three ( 13 per cent) did not state the size of the organisation or the size was not applicable. ${ }^{3}$ Interviews were semistructured - that is, grouped around key themes but open-ended - to avoid constraining participants' possible views. All interviews were coded using computer-aided analysis (NVIVO 10) to identify common themes and challenges, and the three barriers to accountability outlined below emerged from this analysis.

2 Some NFPs use the term 'director' to designate the senior executive officer, who then reports to a management committee or board. To avoid confusion, all participants in the most senior executive role are termed CEO, while 'director' refers only to board members.

3 The typology of large, medium and small NFPs is from the Australian Charities and Notfor-profits Commission (ACNC). To compare this breakdown with the composition of the NFP sector in Australia more broadly, see Knight and Gilchrist (2014). As Knight and Gilchrist (2014) highlight, while the majority of NFPs are small, a core of larger organisations collects most revenue. 


\section{Three potential barriers to accountability for service quality}

NFP staff and directors in this study most strongly endorsed being held accountable for their organisations' capacity to deliver its key service or mission:

I think that society as a whole has become a bit more litigious and accountable ... But I see some of those accountabilities as being a positive thing; I don't think just anybody can educate children. So this level of accountability moves us away from inappropriate people looking after and caring for and educating young children. (Participant [P] 6, CEO, medium-sized childhood education NFP)

There's not a lot of trust in delivery of funding and in flexible use of funding and reporting that appropriately. So I think then what happens is that people think that they'll use that reporting as a kind of lever to make everybody do what they want to do instead of allowing some level of autonomy. But I don't think there should be autonomy in things like regulations and standards for education care settings. That can't be compromised and it's not a burden. It's what people do with it that makes it a burden. (P13, CEO, large childhood education NFP)

Both statements above support external accountability mechanisms that enforce service quality, even while recognising that not all accountability demands serve this end. Since many NFP directors and staff join their organisation to serve its particular mission (see, for example, Light 2002: 109), their commitment to service quality is not surprising. However, recognising that many NFP staff are committed to being accountable for service quality does frame NFP accountability differently to the mainstream governance literature. This literature largely represents accountability as being concerned with managing conflicts of interests - paradigmatically, compelling for-profit managers to serve shareholders' interests (for example, Fama and Jensen 1983a, 1983b; see also Brennan and Solomon 2008; Olson 2000). Of course, NFPs also face conflicts of interests, especially since they are often accountable to multiple stakeholders (Ebrahim 2003; O'Dwyer 2005), and also are susceptible to fraud (BDO 2014). Yet the focus of NFP staff and directors on being accountable for service quality suggests that NFP accountability can also break down in different ways. In particular, the three barriers explored below are 
predominately cases where people intend to be accountable for service quality, but various reporting, governance or regulatory practices create impediments.

\section{Barrier one: Reporting that is weakly linked to service quality}

Since most participant NFPs are highly dependent on external funding, what NFPs report is strongly influenced by funders' actual or perceived reporting requirements:

Our focus is on being accountable to the government department that let the contract and gave us the money. (P5, director, large social services NFP)

Interviewer: Are there particular groups that figure in those [board] conversations, as these are the people we really need to be concerned about?

P1: Yes, it's organisations so it's always funders. (P1, director, large social services NFP)

P12: All we measure at the moment is activities. You know, the number of people in beds on a particular night, etc.

Interviewer: Those kinds of things, are they being measured because that's what ...

P12: Government requires. (P12, manager, large social services NFP)

It has been widely observed that reporting and regulatory requirements can sap NFPs' limited time and resources (for example, Productivity Commission 2010); however, participants in this study were not primarily concerned about time spent reporting as such. Rather, participants were predominantly concerned with reporting that did not adequately measure or evaluate the quality or impact of their services, in at least three interrelated respects.

The first was reporting to funders that did not ask for enough information to genuinely assess a program's quality or impact:

This is the only program that I've ever worked in where the reporting is so minimal. And accountability - incredibly — almost non-existent ... When I first started I thought it was like any other government contract and I was logging everything that I was doing and they came in and said there is too much in there. Stop it. Just key actions or key progress... 
If we had those things in place it would probably be a bit better because it seems like whilst we're doing such minimal reporting it feels as though the reporting that we do has no value whatsoever. (P14, manager, large community development NFP)

To be clear, this quote does not reflect all participants' experiences; many have stringent and extensive reporting requirements. It does, however, speak to the variability of funders' reporting requirements, which range from very detailed to almost non-existent for even multimillion-dollar programs. The quote above also indicates how too little reporting can sometimes be a greater imposition on NFPs than more rigorous reporting. Poor-quality reporting still takes time and resources, but may not deliver useful information:

[M]aybe this [lack of detailed reporting] has something to do with why the program hasn't been funded beyond this year ... When you look at the success of the program it's very hard to see where the success is because it hasn't been evaluated, no-one has been held accountable. Some organisations are doing really fantastic things and some are really, really poor. (P14)

This quote illustrates how rigorous reporting on service quality not only enables funders to discharge their oversight role, but also may have more tangible value to NFPs by enabling them to demonstrate the value their services deliver.

A second way reporting to funders became detached from service quality was when measured outputs were weakly linked to service outcomes:

At the moment all we do is measure how many people come in and how many supports we give them. That doesn't tell us if we're actually doing anything that has any lasting change. (P12)

Everyone likes the idea of results-based accountability-it's just how you actually do [it] that is important. (P15, service worker, large community development NFP)

These quotes highlight the continued reliance on measuring outputs despite several decades of discussion about more outcomes-based measures (for example, Plantz et al. 1997; Kaplan 2001). However, they also point towards the ongoing difficulties of using alternative measures, as one director explained: 
We have all these program reports that come up that we've done this and we've done that and here are our stats for this month. Here's the cafés income and stuff. Does that fundamentally tell me whether we're doing a good job or not? I don't really know ... I do think that compliance starts being the easy thing to focus on because you can't quite work out how to have a conversation about whether we're doing something well or not. (P1)

This quote suggests that boards themselves are not necessarily aware of how best to measure their organisations' outcomes. There can also be external structural impediments to measuring outcomes, such as the need to demonstrate some results to funders even if these results are weakly linked to the program's ultimate goals:

We still need to have a minimum amount of quantitative [measures] because ... it still provides us with that base because it takes a long time to get outcomes. So those quantitative measures are usually outputs, activities and we need to show that we've done something with the money, not wait 10 years when there are some outcomes from it. Because it can take that long for real sustainable measurable outcomes from a continuous improvement approach. (P12)

This implies that a potential barrier to adopting more outcomesbased measures is providing results that are compatible with short to medium-term funding cycles.

A third way reporting became detached from service quality was when NFPs received little feedback on, or oversight of, the information they reported. Feedback can enable NFPs to use the information they report to assess and improve their services. On this basis, several participants strongly supported even extensive external evaluation of their services provided that funders or evaluators also returned the information and analysis to them:

P14: As part of that [external funding] they actually employ external evaluators to assess, which makes it so much easier because all the reporting is up to them.

Interviewer: So that's positive for you, is it, having external assessors? P14: Yes ... because they're people that are trained in that area, so it's really great data that you get back. (P14) 
In discussing the lack of oversight of NFP reporting, the term 'oversight' refers to funders or other agencies with capacity to investigate and intervene when service providers are not meeting reasonable standards. Many NFP participants in this study supported strong oversight of their sector's services, either because they are committed to their particular service sector (for example, ensuring all children receive a quality education) or because they had a broader interest in ensuring that all providers were effectively using public funds. Several participants reported less external oversight than they would like:

They [the funder] don't seem to be auditing us regularly ... There were members of departments turning up and questioning us and I think that's died off over the last three years ... So we're concerned that we're not delivering the programs as best we can because there's no apparent measurement of it outside the systems I described that we have set up ourselves ...

I'm a taxpayer. I'd like to know that my money's being spent properly ... I'm on the board of the organisation and I don't really know how good our delivery of that [service] is. I'm sure that government doesn't know. (P5)

As discussed further below, these findings suggest that many NFP sector directors and staff may welcome reporting that is focused on service outcomes - and that is properly designed, monitored and enforced, rather than perceiving such reporting as an onerous imposition.

\section{Barrier two: Boards without informed and active members}

A second potential barrier to accountability is when boards lack oversight of, or feedback on, their own performance. This issue was most apparent in member-based organisations whose boards are nominally elected by, and report to, a broader membership. Prior research has identified 'democratic accountability' of elected boards to members as one foundation of NFP legitimacy (Edwards and Hulme 1996). In Australia, Governance Standard 2 issued by the ACNC establishes 'accountability to members' as a minimum requirement of registered charities: 'Charities must be open and accountable to their members. This standard allows members to be in a position to 
understand their charity's operations and raise questions they may have about its governance (for example, about where future activities will be focussed and its financial position)' (ACNC 2013: 6).

Several member-based organisations in this study either had no active members or lacked a membership base that was engaged and knowledgeable enough to question either the board's decisions or the operational and strategic guidance the board provides:

We call a meeting, we're flat out getting half of them to turn up ... We've got to personally invite all our members to come and only half of them turn up and it's probably only for the cup of tea ...

The membership aren't into making sure we're doing our job, like shareholders, perhaps, in a commercial operation or something like that. I'm not quite sure who we report to, really, from a point of view of somebody else keeping an eye on us and making sure that we're doing the jobs. (P5)

P11: Our membership is fairly small ... The members can go to the annual general meeting where the board is elected ...

Interviewer: Does that get much of a turnout?

P11: I don't think it's huge, no. Otherwise, they get a copy of the annual report. Apart from that, I don't know that they have a huge involvement. (P11, senior staff member, social services NFP)

In several cases, the board was largely self-perpetuating. Since the board's operation can affect NFP performance as well as governance (Ranson et al. 2005), the broader issue these cases raise is the extent to which NFP boards have a clear sense of to whom they are accountable and how this accountability is discharged:

I think the board thinks it's accountable for itself or to the chairperson. I don't know that it feels accountable to the client base, but I don't think we're evaluated by the client base ...

The effectiveness of the board is not measured. I can't see how it is measured ... Are any boards effectively measured? (P18, director, large social services NFP) 
Ideally, an active membership should prompt boards to review and evaluate their own performance. In the absence of such a membership, a key question is who is able to ask NFP boards the 'tough questions' about their processes and strategy that they might not have thought of themselves?

\section{Barrier three: Maintaining minimum standards while reducing red tape}

The third potential barrier is maintaining minimum service standards across the sectors NFPs service while also reducing regulatory red tape. In Australia, this potential barrier is linked to the recent debate about whether the ACNC should be retained or disbanded. Paradoxically, both critics and supporters of the ACNC have cited reducing red tape as a primary motivation. Critics have argued that the ACNC adds an unnecessary layer of bureaucracy to the NFP sector (for example, Rittelmeyer 2014). Yet the ACNC's proponents have observed that the commission has reducing red tape as one of its three main mandates, and has repeatedly emphasised that reducing red tape for NFPs is a priority (ACNC 2014; Ernst \& Young 2014).

Amid the underlying consensus about the importance of eliminating red tape, several participants in this study were concerned that the red tape reduction agenda might undermine minimum service standards, especially in service areas such as child care that have especially vulnerable clients:

I know a lot of people say that there's too much red tape in child care but if you didn't have the red tape then people would take the shortcut. (P9, CEO, medium-sized childcare/education NFP)

My view is that any society, wherever you are, needs some sort of framework and guidelines ... so I have no problem with the regulations being in place at all. The red tape that we constantly hear about is more about the systems. It's not the reg[ulations] themselves. If you look at the reg[ulation]s, a lot of them are common sense. You know, children need so much space. (P17, CEO, large childhood education NFP)

These participants indicate the difficulty of distinguishing red tapepresumably 'bad legislation' or standards - from effective legislation. In their ACNC-sponsored report on red tape, Ernst and Young (2014: 56) state that red tape is: 'Regulatory and reporting obligations 
that are excessive, unnecessary or confusing. Given this definition, determining what constitutes red tape is open to being highly subjective to an individual or organisation and is highly dependent on the unique circumstances of the charity involved' (emphasis added).

The clearest examples of what participants in this study viewed as red tape were duplicative reporting and accreditation requirements. One NFP undertook three separate accreditation processes for essentially the same service. Another participant described the overlapping standards in homelessness services:

We have the National SHS Standards - the National Specialist Homelessness Services Standards - that are still in draft form. You've got the NSW SHS Standards. You've also got the NSW Good Governance. You've got the Queensland Human Service Standards ... You've got all of these standards that are very, very similar and people have got to measure their work against often two or three of them when they're all very similar ... Why can't we just have one set? (P12)

While eliminating duplication is an important and widely shared objective, what constitutes unnecessary regulation is less clear:

What concerns me about this red tape issue is [that] some people refer to it, the red tape, as ... documenting children's learning. We've got a really strong stance that that is a basic teaching tool for any teacher no matter what age group you're working with. That's not the regulatory burden. (P17)

The example of whether or not requiring childhood education providers to document children's learning is red tape illustrates the difficulty of determining when regulation or accreditation standards are unnecessary. To categorise a requirement to document children's learning as red tape necessitates a substantive judgement about whether such documentation improves learning outcomes, and about whether it is possible to enforce this practice. These judgements require expertise in the specific service area - in this case, childhood education - rather than broad-based policy expertise. In essence, participants were concerned that the policy consensus on removing duplicative red tape might facilitate watering down service standards that are deemed 'unnecessary', but without the substantive discussion or debate - especially with sector experts and stakeholders - that more rigorous and responsible reform would require. 
A broader issue here is that the shift towards market-based funding models makes legislated minimum service standards more rather than less important to ensuring sectorwide accountability on service quality. In this study, the participants who were most concerned about the potential for deregulation to decrease service quality were concentrated in sectors facing growing competition from for-profit providers. However, actual or proxy market systems can pressure both for-profit and NFP providers to cut costs. While competitive pressures may lower prices for end users, the risk is that pressures to reduce costs will drive declining service quality if minimum standards are not carefully monitored and actively enforced. This risk is especially serious in core public services such as childhood education and health that also have vulnerable clients and significant - and arguably intractable - information asymmetries between service 'buyers' and 'sellers'.

\section{Addressing barriers to NFP accountability: Prospects and possibilities}

Given the diversity of the services NFPs provide, and the differences in NFP size and structure (see Knight and Gilchrist 2014), there are likely to be many possible responses to the barriers identified above. Moreover, some issues - most notably, distinguishing 'necessary' from 'unnecessary' regulation-require sector-specific expertise rather than generic principles. Nonetheless, participants' own experiences, and relevant academic research, suggest possible responses to each barrier.

\section{Reconnecting reporting to services}

Funders have considerable power to modify their reporting requirements (Oakes and Young 2008), and could more closely link reporting to service outcomes in several practical ways. The most obvious include: 1) requiring sufficiently detailed reporting; 2) including outcomes as well as output measures; and 3) ensuring reporting data are fed back to NFPs. The ACNC has particularly stressed the last point, with one senior regulator arguing that funders and regulators should either assess reports and disclose their findings or remove the reporting requirement: 
If you cannot locate its [the report's] necessity and also then its effectiveness to meet that necessity, you've got an unnecessary piece of [reporting]. So every year someone puts in their association's report to a state regulator, and it's put into that filing cabinet and locked. It's not made available to the public. No-one ever looks at it. There's no assessment of its worth. What is being achieved by filling out that form? Absolutely nothing. (P21, senior regulator)

The study findings also imply, however, that practical improvements in NFP reporting requirements may need a broader shift in how both funders and NFPs conceptualise accountability - namely, a shift from largely formal reporting towards an ongoing relationship of interaction and dialogue. There are various impediments to this transition, such as the constant rotation of contract managers (Ernst \& Young 2014: 53). Nonetheless, the most effective reporting relationships this study found were part of an open and reciprocal discussion between the NFP, their funder and key stakeholders:

We feel that they [the funder] are approachable, that they know what we're going through so we can trust them ... Essentially, we need to talk to each other at the end of the day ... That's one of the biggest things, I'd say: opening up the communication between governments and NFPs. Having a one-off consultation meeting isn't enough. That's not open communication, that's a consultation. (P10, manager, large social services NFP)

This particular NFP held quarterly meetings with key stakeholders that the funder would attend, and the funder encouraged meetings between different providers to discuss shared concerns. This NFP's reporting also included open-ended narrative as well as quantitative metrics, and the NFP was able to negotiate - within boundaries - which performance metrics their clients completed and how. One reason reporting contributed to service quality in this case was the broader relationship of communication and trust between NFP and funder. This suggests that any review of funders' reporting requirements should include and retain NFPs, and also other key stakeholders such as clients, as part of this ongoing process.

While funders have more scope to change the accountability relationship, NFPs may also be able to improve their reporting practices by measuring and disclosing important service outcomes, even when funders do not require this information. Several NFPs in this study had initiated performance metrics to capture aspects of their services' 
value that their funders' reporting templates overlooked. One example was from a counsellor in a neighbourhood centre whose professional body encouraged members to survey and track clients' well-being over their counselling sessions:

The management committee here's not asking me to do these things, the funding body's not, but our peak body's way of thinking [is that] you want to prove to them things that they [providers and funders] haven't even thought of asking ... This is a social justice issue to keep the money where it belongs. (P15)

The peak body that promoted these surveys was clearly advocating continued public funding for the services their members provide. But provided the measures are reliable and rigorously used, these initiatives tend to reinforce rather than challenge public accountability. Thus, while funders' power over NFPs creates a strong responsibility for funders to more closely link reporting to service quality, NFPs may also be able to instigate closer links between reporting and service quality.

\section{Linking boards to members}

According to one senior regulator, board accountability requires that NFP members have opportunities to question the board, but not necessarily that they regularly do so: 'I don't mind if no-one turns up to the annual general meeting [AGM], and I don't mind if anyone asks any questions at the AGM, as long as they have the opportunity to do so' (P21).

This participant noted that two NFP structures, trustees and memberconstituted boards, lack any formal external constituency. However, he argued that NFPs typically have a broader stakeholder communitytermed 'moral stakeholders' - who are committed to the organisation and are likely to intervene in cases of severe governance failure:

[NFPs are] no longer simply a proprietary ownership of shares and money; it is a sense of responsibility to the wider community for delivering public good. The moral stakeholders have a legitimate interest in ensuring that occurs. So they don't need this proprietarythey don't need to hold a share. They may not even need to hold a membership. They are the people around-the stakeholders around - who will intervene if they suspect something is wrong. (P21) 
Clearly, accountability to moral stakeholders requires public transparency mechanisms, such as the ACNC's charities register, because moral stakeholders do not necessarily have access to the same information as formal members.

However, even given sufficient public transparency, moral stakeholders are at best able to set boundaries on NFPs' governance practices, rather than providing ongoing feedback on board performance. Consequently, in the absence of active members, boards need to find processes or practices that perform a comparable function. One possible substitute - albeit imperfect - for an active membership is to more rigorously assess board performance against external best-practice governance standards, which could at least provide an external reference point for boards' decision-making processes. While several standards have been proposed, there is no agreed standard of NFP board best practice, despite board members requesting such standards or guidance: '[What I would like is] to be able to say, here's your best governance guidelines, best practice in the organisation of not-for-profits' (P16, director, large social services NFP).

One large NFP in this study had voluntarily adopted several governance standards and practices from incorporated for-profit organisations, and thereby appropriated an external benchmark to guide their own operation. However, this is unlikely to suit smaller NFPs, and may not apply to other service areas.

A second potential approach is for boards to actively cultivate other stakeholder groups to more regularly question, and provide feedback on, their performance and strategic guidance. P21's concept of moral stakeholders emphasises that NFPs are often located within a broader community who are interested in their activities. The practical challenge is to engage this broader community in more regular discussion of the organisation's direction, and of the board's role in guiding this direction, rather than having moral stakeholders emerge only in organisational crises. External consultants can be used to structure engagement with stakeholders, but this can be both expensive and fraught (O'Dwyer 2005). More regular facilitated meetings with key external stakeholders might play a similar role. 


\section{Red tape and minimum service standards}

Participants' concerns about eliminating red tape reveal a potential tension between removing duplicative and unnecessary regulatory constraints and preserving sector-wide service quality benchmarks. As section two observed, judgements about what service standards are necessary require sector-specific experience and expertise. Inter alia, this suggests that while duplicative red tape might be identified and removed at a national policy level, deciding what constitutes unnecessary service quality regulation or standards will require far-reaching engagement and consultation, including with NFPs who deliver these services and the clients who receive them.

In determining what regulation affects service standards, it is important to recognise how minimum qualifications for NFP staff may enhance accountability as well as improve service quality. In several participant organisations, especially those without a well-informed and experienced board, NFP staff described holding each other accountable to shared professional standards and norms. Professional standards and norms do not replace external reporting and oversight; however, they do illustrate how minimum staff qualifications may also support workplace-level accountability. At the same time, staff qualification requirements are attracting close scrutiny due to tighter public budgets and more market-based funding, both of which can pressure organisations to employ staff who are less qualified and therefore cheaper to employ. While the merits of mandating particular minimum qualifications would need to be addressed at the servicesector level, this study suggests that it would be misleading to debate these minimum qualification standards solely within a red tape framework. Rather, since professional qualifications can also support accountable service delivery, regulated minimum qualifications are part of a broader debate about the right balance between accountability and cost.

\section{Conclusion}

This chapter has reviewed three potential barriers to NFPs being accountable for the quality or impact of their services. First, NFPs' reporting to funders may not provide sufficient information or feedback on the quality or impact of their services. In response, 
the chapter suggests not only that funders may need to adapt their reporting requirements, but also that NFP reporting may need to be set within a more open and ongoing dialogue between NFPs, funders and other stakeholders. Second, while board accountability to members is central to governance theory and standards, many NFP boards lack members who can effectively question their performance. The challenge this poses to NFPs' boards is to introduce external standards or relationships that perform a comparable function. Third, the chapter illustrates how agendas to reduce red tape could impede sectorlevel accountability if they undermine minimum service standards, especially in increasingly cost-sensitive funding environments. To mitigate this risk, red tape reduction that bears on service standards will need extensive sector-level engagement. More generally, while the shift towards more market-based funding systems can expand client choice, it also makes it more important to carefully preserve minimum service standards and qualifications against inevitable costdriven critiques.

\section{References}

Australian Charities and Not-for-profit Commission (ACNC). 2013. ACNC Governance Standards Guidance. Melbourne: Australian Government and ACNC.

Australian Charities and Not-for-profit Commission (ACNC). 2014. Forum Report: Measuring and reducing red-tape in the not-for-profit sector. Melbourne: Australian Government and ACNC.

BDO. 2014. BDO Not-for-Profit Fraud Survey 2014. Sydney: BDO Australia Limited.

Brennan, N. and J. Solomon. 2008. 'Corporate governance, accountability, and mechanisms of accountability: An overview.' Accounting, Auditing and Accountability 21(7): 885-906.

Carnegie, G. D. and P. W. Wolnizer. 1996. 'Enabling accountability in museums.' Accounting, Auditing \& Accountability Journal 9(5): 84-99.

Ebrahim, A. 2003. 'Accountability in practice: Mechanisms for NGOs.' World Development 31(5): 813-29. 
Edwards, M. and D. Hulme. 1996. 'Too close for comfort? The impact of official aid on nongovernmental organizations.' World Development 24(6): 961-73.

Ernst \& Young. 2014. Research into Commonwealth regulatory and reporting burdens on the charity sector. A Report prepared for the Australian Charities and Not-for-profits Commission. Ernst \& Young, Canberra.

Fama, E. F. and M. C. Jensen. 1983a. 'Agency problems and residual claims.' Journal of Law and Economics 26(2): 327-49.

Fama, E. F. and M. C. Jensen. 1983b. 'Separation of ownership and control.' Journal of Law and Economics 26(2): 301-25.

Fox, J. A. and L. D. Brown (eds). 1998. The Struggle for Accountability: The World Bank, NGOs and grassroots movements. Cambridge, Mass.: The MIT Press.

Gray, R. 1983. 'Accounting, financial reporting and not-for-profit organisations.' AUTA Review 15(1): 3-23.

Kaplan, R. S. 2001. 'Strategic performance measurement and management in nonprofit organizations.' Nonprofit Management and Leadership 11(3): 353-70.

Knight, P. A. and D. J. Gilchrist. 2014. Australian Charities 2013: The first report on charities registered with the Australian Charities and Not-for-profits Commission. Melbourne: ACNC.

Light, P. C. 2002. Pathways to Nonprofit Excellence. Washington, DC: The Brookings Institution Press.

Oakes, L. S. and J. J. Young. 2008. 'Accountability re-examined: Evidence from Hull House.' Accounting, Auditing \& Accountability Journal 21(6): 765-90.

O'Dwyer, B. 2005. 'The construction of a social account: A case study in an overseas aid agency.' Accounting, Organizations and Society 30(3): 279-96.

Olson, D. E. 2000. 'Agency theory in the not-for-profit sector: Its role at independent colleges.' Nonprofit and Voluntary Sector Quarterly 29(2): 280-96. 
Plantz, M. C., M. T. Greenway and M. Hendricks. 1997. 'Outcome measurement: Showing results in the nonprofit sector.' New Directions for Evaluation 1997(75): 15-30.

Productivity Commission. 2010. Contribution of the Not-for-Profit Sector. Canberra: Productivity Commission. Available from: pc.gov. au/inquiries/completed/not-for-profit (accessed 14 August 2014).

Ranson, S., C. Farrell, N. Peim and P. Smith. 2005. 'Does governance matter for school improvement?' School Effectiveness and School Improvement 16(3): 305-25.

Rittelmeyer, H. 2014. Independent Charities, Independent Regulators: The future of not-for-profit regulation. Sydney: Centre for Independent Studies.

Roberts, J. and R. Scapens. 1985. Accounting systems and systems of accountability: Understanding accounting practices in their organisation context.' Accounting, Organizations and Society 10(4): 443-56.

Sinclair, A. 1995. 'The chameleon of accountability: Forms and discourses.' Accounting, Organizations and Society 20(2-3): 219-37.

Stewart, J. 1984. 'The role of information in public accountability.' In Issues in Public Sector Accounting, eds A. Hopwood and C. Tomkins, 13-34. Oxford: Philip Allan.

Treasury. 2011. Scoping Study for a National Not-for-Profit Regulator. Canberra: Australian Government. Available from: archive.treasury. gov.au/contentitem.asp?ContentID=2054 (accessed 14 August 2014).

Unerman, J. and B. O'Dwyer. 2006. 'Theorising accountability for NGO advocacy.' Accounting, Auditing \& Accountability Journal 19(3): 349-76. 
This text is taken from The Three Sector Solution: Delivering public policy in collaboration with not-for-profits and business, edited by John Butcher and David Gilchrist, published 2016 by ANU Press, The Australian National University, Canberra, Australia. 